\title{
The Potency of Yellow Fever Vaccines Sold in Open Markets in Eastern Nigeria
}

\author{
Godwin Chukwuma, Linda Okoye, Kenneth Ngwoke* and Charles Esimone
}

Nnamdi Azikiwe University, Nigeria

\begin{abstract}
Background: There has been a re-emergence of Yellow Fever (YF) disease across Africa and in South America since the 1980s. According to WHO estimate, 508 million people in 32 countries are at risk because of their location in the rainforest region, the habitat of the vector mosquito.Intense YF virus circulation occurred in West Africa in 2008 and 2009 with outbreaks reported from Burkina Faso, Cameroon, Central African Republic, Chad, Congo, Côte d'Ivoire, Guinea, Liberia and Sierra Leone. Between January 2010 and March 2011, outbreaks of YF were reported to the WHO by Cameroon, Côte d'Ivoire, Democratic Republic of Congo, Sierra Leone, Senegal and Uganda. Most recent outbreaks in Africa were reported simultaneously in Ghanaand Cameroun on the 3rd of February, 2012. This study investigates a possible correlation between vaccine manufacture, cold chain handling and vaccine potency/ stability as a risk factor in the repeated outbreak of yellow fever in the face of increased immunisation coverage in Nigeria and West Africa by extension.
\end{abstract}

Methods: Samples of Stamari ${ }^{\circledR}$, a yellow fever vaccine collected from different open market sources were subjected to potency test by measuring their immunogenicity indicators. Their embryo infectivity dose and mice $L_{50}$ as well as their pyrogenicity were determined.

Results: Results showed that $42 \%$ of the vaccines tested were not suitable for use even though the vaccine vial monitor certifies they are fit for use.

Conclusion: A large proportion of YF vaccines sold in open markets in Eastern Nigeria may be compromised in terms of potency. Vaccine vial monitors only are not entirely reliable as indicators of vaccine potency or usability.

Keywords: Vaccine; Vaccine Vial Monitor; Yellow fever; $\operatorname{EID}_{50}$; Yellow fever vaccine

\section{Introduction}

Yellow Fever (YF) is a viral haemorrhagic fever characterised by fever, chills, headache and backache, myalgia, nausea, vomiting, facial flushing, and conjunctival injectionat the onset and may result to death if symptoms persist. Yellow Fever virus was first isolated in 1927 by French and American researchers in Africa [1]. The Asibi strain was isolated in Ghana, West Africa while the French viscerotropic virus strain was isolated from a Syrian man, Françoise Mayali, in Senegal. The first South American strain, JSS, was isolated in Brazil in 1935 [1,2]. Eradication and control of yellow fever has remained problematic in West Africa and South American regions because of the prevalence of rainforest habitat of the vector mosquitos in these regions.

During the 1930s both of these wild-type strains were used to empirically derive live attenuated vaccines known as the French neurotropic vaccine and $17 \mathrm{D}$, respectively. Only one (17D vaccine) of the two live attenuated vaccines developed in the 1930s is still in use today. The second (French neurotropic vaccine) was discontinued due to high rate of fatalities resulting from encephalities [3]. The use of these vaccines aided in the control and reduction YF morbidity and mortality in the endemic regions.

However, there has been a re-emergence of yellow fever disease across Africa and in South America since the 1980s. A total of 18,735 yellow fever cases and 4,522 deaths reported from 1987 to 1991 represents the greatest amount of yellow fever activity reported to the WHO for any 5-year period since 1948 [4]. According to WHO estimate, 508 million people in 32 countries are at risk because of their location in the rainforest region, the habitat of the vector mosquito. Intense YF virus circulation occurred in West Africa in 2008 and
2009 with outbreaks reported from Burkina Faso, Cameroon, Central African Republic, Chad, Congo, Côte d'Ivoire, Guinea, Liberia and Sierra Leone ${ }^{4}$. Between January 2010 and March 2011, outbreaks of YF were reported to the WHO by Cameroon, Côte d'Ivoire, Democratic Republic of Congo, Sierra Leone, Senegal and Uganda. Most recent outbreaks in Africa were reported simultaneously in Ghana and and Cameroun on the 3rd of February, 2012 [5].

Nigeria, a West African country situated in the yellow fever zone has since 1990s added YF vaccine to its Expanded Programme on Immunization (EPI) programme due to repeated outbreaks and the need to increase the vaccination coverage in the country which has been reported to be one of the lowest in the world [6]. Although the last reported outbreak in Nigeria was in 2000, there is a need to take a look at the potency and stability of the vaccines used for national immunisation. This is because most part of West Africa is located within the equator where the temperature can remain above $37^{\circ} \mathrm{C}$ for a considerable part of the year such that stability and stability monitoring method should be re-evaluated in the light ofrecurrent outbreaks in the regions to ensure that people are vaccinated with vaccines that have the required minimum level of immunogenicity.

${ }^{*}$ Corresponding author: Kenneth Ngwoke, Nnamdi Azikiwe University, Nigeria, Tel: 2347051403916; E-mail: kg.ngwoke@unizik.edu.ng

Received October 22, 2013; Accepted November 23, 2013; Published November 26, 2013

Citation: Chukwuma G, Okoye L, Ngwoke K, Esimone C (2013) The Potency of Yellow Fever Vaccines Sold in Open Markets in Eastern Nigeria. J Bioanal Biomed 5: 133-137. doi:10.4172/1948-593X.1000094

Copyright: (c) 2013 Chukwuma G, et al. This is an open-access article distributed under the terms of the Creative Commons Attribution License, which permits unrestricted use, distribution, and reproduction in any medium, provided the original author and source are credited. 
The stability of the yellow fever (YF) vaccines in storage has been a matter of concern. Most of them lose some activity during storage at right temperature and they deteriorate quite quickly at higher temperatures $[7,8]$. Even with the introduction of vaccines with enhanced stability by several manufacturers, the result of stability studies of 11 YF vaccines sent to WHO by manufacturers showed a wide variation in stability among vaccines. The number of days required to reduce the initial titre to 1000 infective units when vaccines were kept at $37^{\circ} \mathrm{C}$ ranged from 1 to 5 days in four vaccines, from 13 to 21 days in two vaccines, and from 38 to 146 days in five vaccines. This is a significant level of variability that calls for a strict cold chain handling adherence.

A vaccine vial monitor (VVM) was introduced as an indicator of heat induced deterioration in vaccines. Vaccine vial monitors were introduced for use on vaccine vials in 1996 to enable health workers assess whether vaccine in individual vials has been exposed to too much heat over time. Vaccine vial monitors, which measure exposure to heat, are time- and temperature sensitive labels attached to vials of vaccine at the time of manufacture [9]. Through a gradual colour change they warn health workers and storekeepers when a vaccine has been excessively exposed to heat and should not be used. They are designed to meet the vaccine's heat stability curve, allowing a margin of safety [10]. They comply either with WHO requirements for heat stability or with heat stability data provided by the vaccine manufacturer if these are more stringent. If the inner square is a lighter colour than the outer reference ring then the vaccine can be used. If the inner square is the same colour as the outer ring or darker than it, then the vaccine should not be used. However, the suitability of this monitoring method has been put to question by the result of stability studies of 11 YF vaccines sent to WHO by manufacturersmentioned above which suggests that vaccines can detriorate even when they are not exposed to heat for a long time as was shown in the report above.

This study investigated the correlation between vaccine manufacture, storage and cold chain handling and vaccine potency/ stability as a risk factor in the repeated outbreak of yellow fever in the face of increased immunisation coverage in Nigeria and West Africa by extension.

\section{Material and Methods}

\section{Vaccine samples}

Four samples of lyophilized yellow fever vaccines Stamaril'(Sanofi Pasteur, France)supplied with diluents were obtained from each of the three major open markets in South-eastern Nigeria namely: bridge head market [Onitsha, Anambra State (Lot number- G5320)], Ariaria main market [Aba, Abia State (Lot number-G5424)] and Ogbete main market [Enugu, Enugu State (Lot number G5320)]. The Inner Square in the Vaccine Vial Monitor (VVM) was lighter than the outer ring indicating that it was viable and can be used. During the transportation to the laboratory, the vaccines were stored in an ice pack to maintain the cold chain sequence before transferring to regulated refrigerator in the Laboratory mainatained at $4^{\circ} \mathrm{C}$ prior to use.

\section{Pre- incubated 9 days old embryonated chicken eggs}

Pre- incubated 9 dayold embryonated chicken eggs were collected from Arroma farms Nig. ltd, Awka, Anambra state, Nigeria. The viability of the 9-day old embryonated eggs was determined under a candling lamp, where the embryo appeared as a dark shadow with the head as a dark spot. The inoculation sites were marked.

\section{Mice}

Suckling Swiss albino mice not more than 6 days old with an average weight of $2.5 \mathrm{~g}$ were collected from Faculty of Pharmaceutical Sciences, NnamdiAzikiwe University Agulu Campus Animal house. Animals were handled strictly in accordance with EU directive 2010/63/EU on the protection and handling of animals used for scientific purposes.

\section{Preparation of washed chicken red blood cells (RBCP)}

The chickens that were used for the work were housed separately from other fowls in the animal house. Blood was collected from the wing vein of the chicken, using syringes that already contain 0.33 $\mathrm{mL}$ of Acid Citrate Dextrose, ACD (BDH, England) per mililitre of blood. The blood was centrifuged at 3,000 rpm for 5 minutes; this was done repeatedly until the supernatant became clear, discarding the supernatant and topping the sediment with ACD in each cycle. The supernatant was finally discarded leaving behind a clear, washed RBC. Thereafter, $2 \%$ suspension of RBC was prepared from the washed RBC by mixing $0.2 \mathrm{ml}$ of the mixed and properly washed RBC with $9.8 \mathrm{ml}$ of PBS.

\section{Preparation of inoculum and dilutions}

The lyophilized vaccines were reconstituted with the company supplied diluent (buffered saline solution) to a total volume of $5 \mathrm{ml}$ according to manufacturer's directive. Two-fold serial dilutions of the vaccine were carried out into three places using PBS as the diluent. Each dilution was labelled and kept in an ice packed container and used within one hour after the initial reconstitution.

\section{Heamaglutination test: inoculation of the allantoic cavity}

The candled eggs were swabbed with $70 \%$ alcohol. A sterile egg hole punch was used to make a hole in the egg at the marked inoculation site. Using a $1 \mathrm{~mL}$ syringe, $0.2 \mathrm{ml}$ of the reconstituted vaccine was drawn and injected slowly into the egg. Keeping the needle and syringe vertical, the needle was passed through the hole in the eggshell penetrating approximately $16 \mathrm{~mm}$ into the each egg to reach the allantoic cavity. Five embryonated eggs were inoculated with each dilution using separate syringes and needles. The egg hole in the shell after inoculation was sealed with melted wax.

The inoculated eggs were returned back to the incubator, and the temperature and humidity of incubator adjusted to $37^{\circ} \mathrm{C}$. The eggs were incubated for four days and turned four times daily and candled each day to determine their viability. After day one all dead eggs were discarded.

After incubation, the eggs were removed from the incubator and chilled overnight in a refrigerator at $4^{\circ} \mathrm{C}$ and assayed using Allan and Gough (1974) method [11]. Everything was carried out in an aseptic condition in a Class II laminar flow chamber.

\section{Determination of the $50 \%$ embryo infectivity dose $\left(\mathrm{EID}_{50}\right)$}

The data generated from the haemagglutination assay was computed and used to calculate the $\operatorname{EID}_{50}$ using Reed and Muench Technique [12]. The aim was to measure the concentration of Yellow fever virus in the vaccine suspension.

\section{Determination of the $50 \%$ mice lethal dose}

Five suckling Swiss albino mice not more than 6 days old and weighing an average of $2.5 \mathrm{~g}$ were inoculated intracerebrally in the 
mid frontal area with $0.02 \mathrm{ml}$ of each dilution, using a separate syringe and needle for each inoculation. A total of fifteen mice were used for each vaccine analysed. The mice were observed for twenty one days as stipulated in the European pharmacopeia for encephalitis and death.

At the end of the twenty one days, the number of dead mice and those that survived were used to calculate the lethal dose using Reed and Muench technique [13]. The data generated was used to calculate the $50 \%$ lethal dose.

\section{Endotoxin concentration testing}

This was determined by the LAL gel clot endpoint assay. This test was carried out in a laminar flow cabinet and all glassware used was depyrogenated in a thermo lab hot air oven at a temperature of $250^{\circ} \mathrm{C}$ for 2 hours. In other to determine the endotoxin load of the various yellow fever vaccines, 8 -fold serial dilutions of the vaccine were carried out into 8 places using LAL reagent water. This was followed by the reconstitution of endotoxin raised from $E$. coli (055:B5)(Lonza, USA) with $5.0 \mathrm{ml}$ of LAL reagent water (Lonza, USA) to give a $20 \mathrm{EU} / \mathrm{ml}$ endotoxin solution which was vortexed for $15 \mathrm{~min}$. The solution was further diluted to give a $1 \mathrm{EU} / \mathrm{ml}$ solution. Two-fold serial dilution of the $1 \mathrm{EU} / \mathrm{ml}$ endotoxin solution was prepared in LAL water to bracket the lysate sensitivity. Each dilution was vortexed for 1 minute before proceeding to the next dilution. The lysate was reconstituted with 5.2 $\mathrm{ml}$ of LAL reagent water and swirled gently to avoid destruction of the protein. Avoiding endotoxin contamination, $0.1 \mathrm{ml}$ of each dilution of the different yellow fever vaccines were transferred into a pyrogen-free reaction tube followed by the addition of $0.1 \mathrm{ml}$ of the reconstituted lysate.

The content was mixed thoroughly and the tube placed in an incubating block for 1 hour at $37^{\circ} \mathrm{C} \pm 1^{\circ} \mathrm{C}$. This procedure was repeated for each dilution of endotoxin. The Yellow fever vaccine samples were assayed in parallel with a control standard endotoxin $[(\mathrm{PPC}=\mathrm{CSE}+$ Lysate+vaccines $), \quad($ Test $=$ Lysate + Vaccine $)+\mathrm{NPC}=\mathrm{LAL}$ water+Lysate)]. After incubation, the result was taken by carefully removing the assay tubes and inverting to $180^{\circ} \mathrm{C}$. A positive case or result is identified by the formation of firm gel clot that remains intact momentarily when the tube is inverted, while a negative case or result is identified by the absence of gel clot after inversion of tube. Duplicates were included for all the yellow fever vaccine dilutions and endotoxin dilutions. The quantity of endotoxin present in the yellow fever vaccines was calculated by taking the product of denominator of the endpoint dilution fraction and the Lysate sensitivity.

\section{Statistical Analysis}

The difference between the $\mathrm{EID}_{50}$ and the $\mathrm{LD}_{50}$ results of the vaccines were tested at $95 \%$ confidence level with reference to their point of purchase and their mean titre compared to the WHO recommended vaccine standards using the Analysis of Variance (ANOVA) and $\mathrm{P}$ value used to compare the results.

\section{Results and Discussion}

None of the twelve samples was suitable for use based on WHO $\mathrm{LD}_{50}$ specification (Figures 1, 2, 3 and 5) while fourty-two percent of the 12 vials of Stamaril tested for poteny and suitability for use fell short of WHO requirement according to the result of the infectivity titre test (Figure 4).

Only two and one out of the 4 vials sampled each from Onitsha and Aba respectively complied with the regulatory requirement of $\geq$
$3 \log 10$ IU (Figure 4). Conversely, the infectivity titre of vials from Enugu fell above the minimum titre yellow fever virus.However the corresponding $\mathrm{LD}_{50}$ titres $($ mean $=3.53 \log 10)$ were below the required level of activity in this study (Figure 5). The median EID $_{50}$ titre of vaccines gotten from Aba was $2.85 \log 10$ which is $1.65 \log 10$ lower than the recommended titre while the median mouse $\mathrm{LD}_{50}$ titre and $\mathrm{EID}_{50}$ of vaccine samples from Onitsha were 2.77 and $2.97 \log 10$ respectively, which were $1.73 \log _{10}$ and $1.53 \log 10$ below the required activity and virus titre respectively on the average and this depicts a depraciation trend.

WHO requires that the potency of yellow fever vaccine meant for use in humans shall not be less than $3.0 \log 10 \mathrm{IU}$ which is equivalent to $4.5 \log 10 \mathrm{LD}_{50}$ at the end of shelf life. This correalation which was established in older report is no longer valid in the present time according to WHO report, therefore the lack of correalation between infectivity titre and mouse $\mathrm{LD}_{50}$, confirmed by ANOVA result does not invalidate this report [14]. The analysis of Variance showed no correlation.

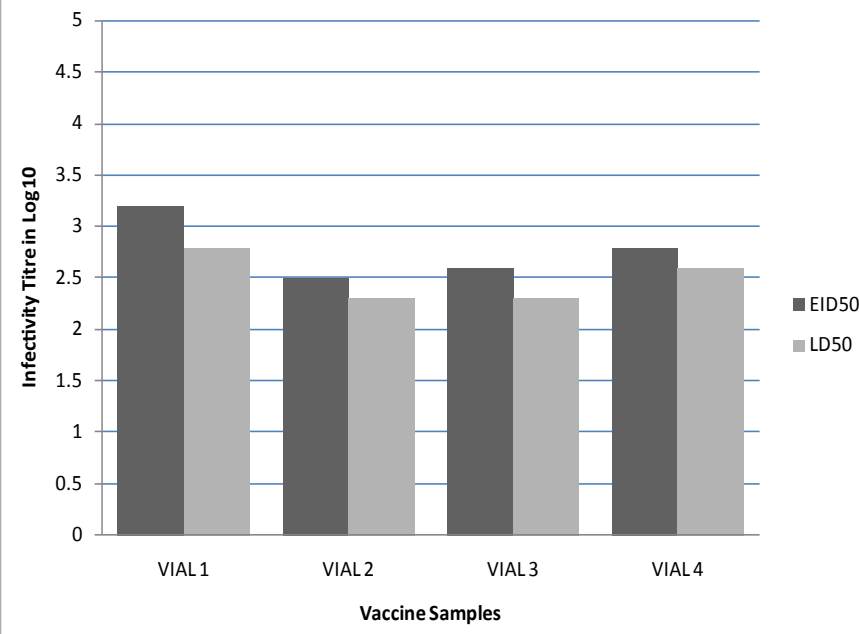

Figure 1: Comparison of $\operatorname{EID}_{50}$ and $\mathrm{LD}_{50}$ of vaccines from $A b a$.

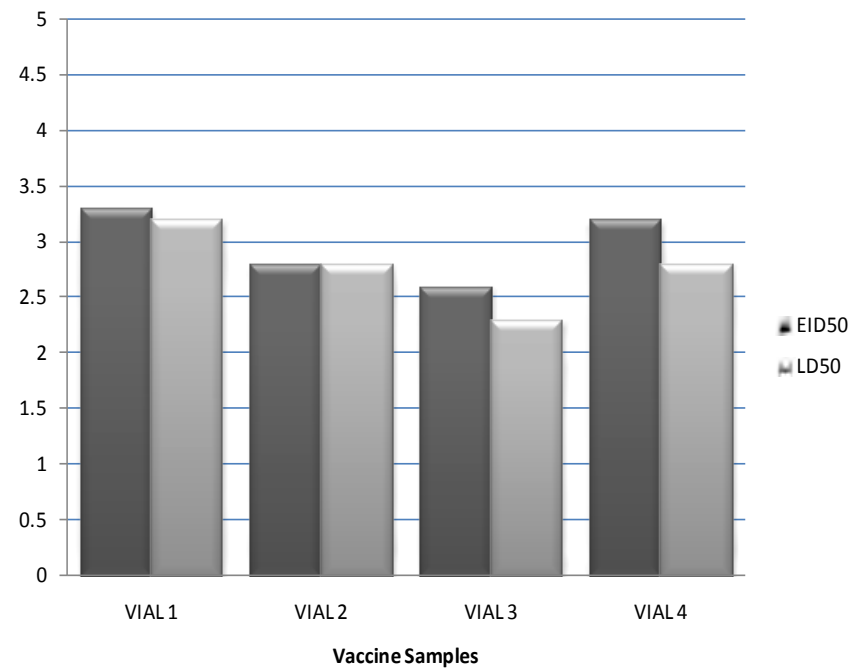

Figure 2: Comparison of EID50 and LD50 of vaccines gotten from Onitsha. 


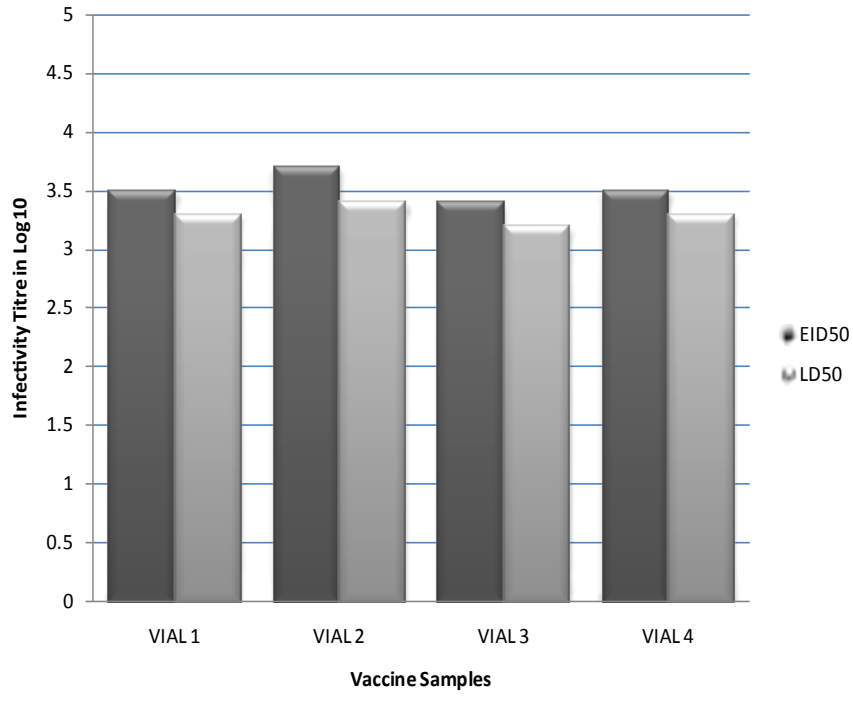

Figure 3: Comparison of $\mathrm{EID}_{50}$ and $\mathrm{LD}_{50}$ of vaccines from Enugu.

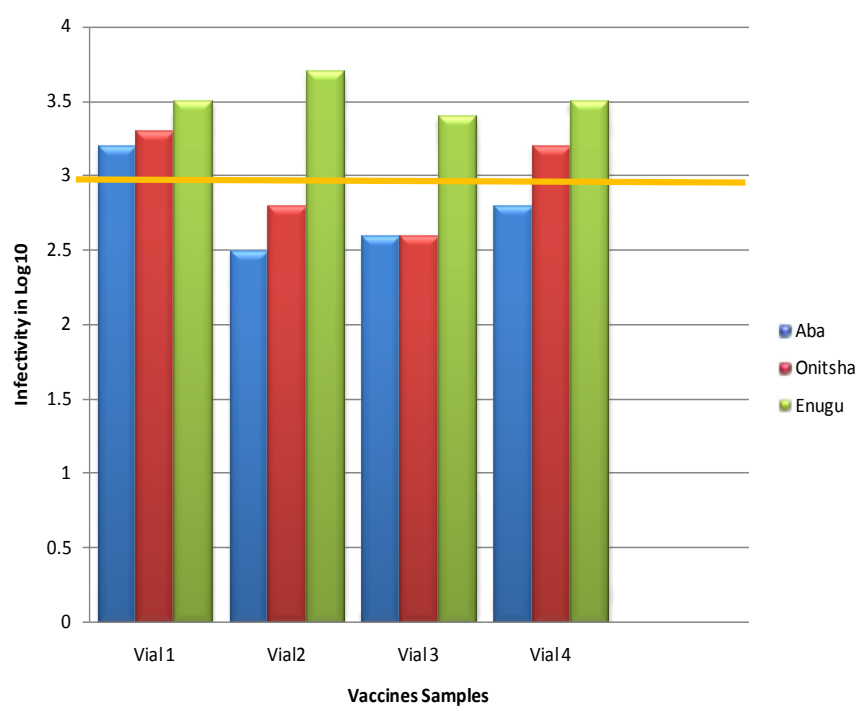

Figure 4: Comparison of the vaccine $\mathrm{EID}_{50}$ according totheir sources.

The report of this work iscorroborated by the result of another work carried out in Osun and Oyo areas of Nigeria. In the report, it was stated that the potencies of yellow fever vaccine collected from Osun and Oyo states of Nigeria were between $0.16 \log 10$ to $0.22 \log 10$ lower than the required level [15].

This therefore implies that the potency ofvaccines sourced from uncontrolled markets in Nigeria tend to flout the minimum immunogenicity requirement and hence are not reliable as sources vaccines for immunization purposes. If vaccines from these markets are used, the implication is that thepopulation immunity will not be proportional to immunization coverage. It will give a false sense of high population immunity which will give rise to defective immunization policy. A potency audit of the vaccines in the national cold rooms and a nationwide potency audit of vaccines sourced from uncontrolled market will be required to get a clearer picture of the potency status of vaccines in Nigeria considering the present and previous reports.
The reason for this depreciation can be attributted to a defect in supply/cold chain management system. If there was no break in the cold chain system and the vaccine is still within its shelf life, then the manufacturing process could be defective. This could only be ascertained at the point of entry.

However, the samples from Onitsha and Enugu have the same lot and batch numbers and by extention were manufactured under identical conditions. It implies that the manufacturing process was not defective.The reason for the low virus titre could therefore be due to stability issue ocassioned by a break in the cold chain resulting to undue exposure to temperatures outside the recommended storage condition of $2-8^{\circ} \mathrm{C}$

Again, the suitability of vaccine vial monitor for monitoring temperature exposure and in extension the stability and usability of a vial is brought into question by this report. This is because the VVM indicated that all the vials were suitable for use based on the colour change comparison while the result of the assay indicates that $42 \%$ of all the vials tested were not suitable for use. This implies that VVM cannot always be relied on solely to determine the suitability and immunogenicity of yellow fever vaccine vials.

The imperfection of VVM lies in the fact that its sensitivity to freezing is not reliable. The VVM strip gets darker during appropriate refrigeration but its colour remains the same when the vaccine is frozen. The temperature range suitable for vaccines storage $\left(2-8^{\circ} \mathrm{C}\right)$ is very narrow because at temperatures below freezing, ice crystals that can denature the proteins or vaccine-adjuvant complex can form while at higher temperature, thermal deterioration will occur. VVM does not monitor abnormal low temperature [14,15] and therefore its use is not adequate. In this case therefore, it is obvious that the low virus titre was due to the storage of the vaccines below freezing temperatures in refrigerators that has no adequate temperature regulator or relaible temperature monitor or both as is obtainable in some shops in Nigeria.

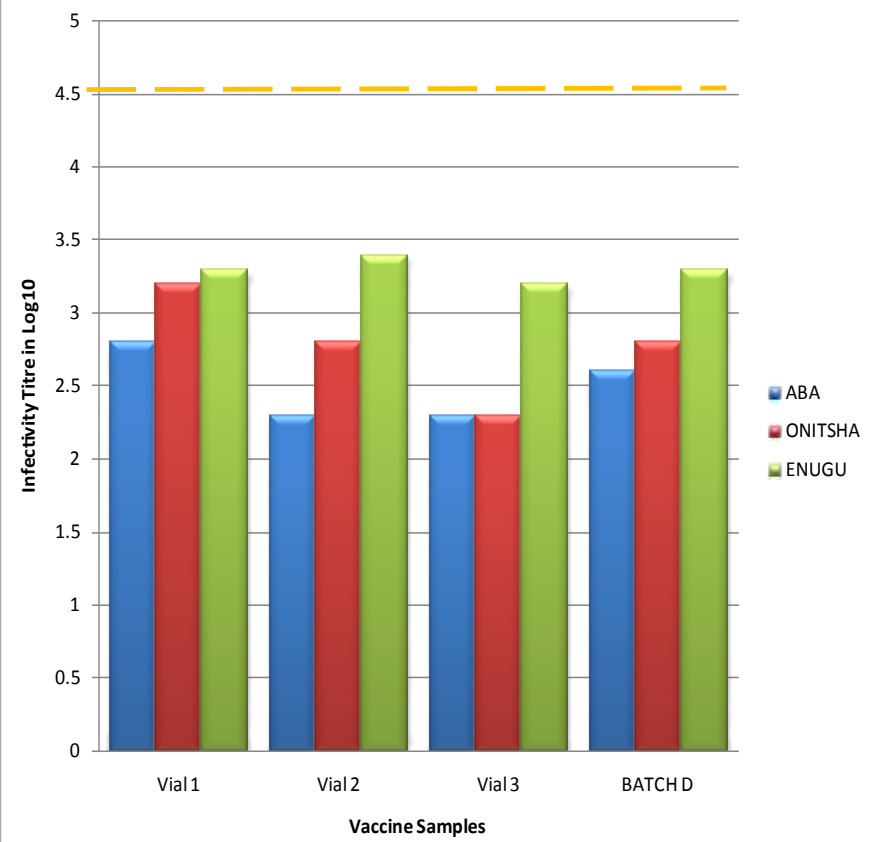

Figure 5: Comparison of the vaccine $\mathrm{LD}_{50}$ according to theirsources. 
Citation: Chukwuma G, Okoye L, Ngwoke K, Esimone C (2013) The Potency of Yellow Fever Vaccines Sold in Open Markets in Eastern Nigeria. J Bioanal Biomed 5: 133-137. doi:10.4172/1948-593X.1000094

As was expected, the LAL test showed that the vaccine vials tested were pyrogen free.

This work makes a good case for adequate control of distribution and supply chain management system as most of the sellers of the vaccines may not be aware of the lower temperature restrictions. Adequate nationwide awareness campaign and seminars for vaccine handlers both in the national cold rooms and in the uncontrolled markets should be carried out to ascertain the viral status of vaccines within the borders of Nigeria.

\section{Conclusion}

A large proportion of YF vaccines sold in open markets in Eastern Nigeria may be compromised in terms of potency. Vaccine vial monitors only are not entirely reliable as indicators of vaccine potency or usability.

\section{References}

1. http://apps.who.int/iris/bitstream/10665/64455/1/WHO_EPI_GEN_98.11.pdf

2. http://whqlibdoc.who.int/hq/1998/WHO_GPV_98.04.pdf

3. Mukhopadhyay S, Kuhn RJ, Rossmann MG (2005) A structural perspective of the flavivirus life cycle. NatRevMicrobiol 3: 13-22.

4. World Health Organization (2011) Yellow fever in Africa and Central and South America, 2008-2009. WklyEpidemiol 86: 25-36.
5. http://www.who.int/csr/don/archive/disease/yellow_fever/en/

6. Cambridge Economic Policy Association, 2010: Global Alliance for Vaccines andlmmunization second evaluation. Country Evaluation Report for Nigeria 209: 132-143.

7. Robin Y (1971) Étude de la thermostabilité du vaccin antiamaril sur des echantillons de huit lots provenant de divers pays. Bull WHO 44: 729-737.

8. Sood DK (1993) Study on the stability of 17D-204 yellow fever vaccine before and after stabilization. Vaccine 11: 1124-1128.

9. http://www.spc.int/phs/pphsn/Outbreak/Thermostablilty_of_Vaccines.pdf

10. Allan WH, Gough RE (1974) A standard haemagglutination inhibition test for Newcastle disease. (1). A comparison of macro and micro methods. Vet Record 95: 147-149.

11. Myers LE, McQuay LJ Hollinger FB (1994) Dilution Assay Statistics. J Clin Microbiol 32: 732-739.

12. http://www.who.int/biologicals/publications/trs/areas/vaccines/yellow fever/ ECBS\%202008\%20WHO_TRS\%20872\%20\%20Amndmt_Yellow\%20Fever. pdf

13. Adu FD, Adedeji AA, Esan JS, Odusanya OG (1996) Live viral vaccine potency: an index for assessing the cold chain system. Pub Health 110: 325-330.

14. Zweig SE (2006) Advances in vaccine stability monitoring technology. Vaccine 24: 5977-5985.

15. Corbel MJ (1996) Reasons for instability of bacterial vaccines. Dev Biol Stand 87: 113-124. 